\title{
Felice Battaglia e l'università del dopoguerra
}

Simona Salustri

ISSN: 2282-4979

DOI: 10.12977/ereview123

Questo articolo è stato sottoposto a un processo di double blind peer review

The essay focuses on Felice Battaglia and his activity at the University of Bologna during the post-war period, without forgetting the role of the professor in the general context of the Italian universities development before 1968.

Felice Battaglia è stato un insigne giurista, un docente di grande cultura, filosofo di fama internazionale, un formatore in grado di incidere su un'intera generazione di intellettuali. Non da ultimo, ha preso parte da protagonista ai più importanti cambiamenti della storia universitaria italiana nel primo ventennio del dopoguerra [Marchello 1953; Matteucci 1977-78; Polato 1989; Scerbo 1990]. All'interno dell'università e delle istituzioni di alta cultura ha ricoperto gli incarichi più prestigiosi dapprima come preside di Facoltà, poi come rettore dell'Università di Bologna (dal 1950-51 fino al 1955-56, poi nuovamente dal 1962-63 al 1968), divenendo membro della Commissione nazionale d'inchiesta per la riforma della scuola, della Consulta didattica nazionale e del Consiglio superiore della Pubblica istruzione [Polato 1988].

In questa sede ci concentreremo prevalentemente su Battaglia nel suo ruolo di rettore, e in modo particolare sui suoi primi due mandati', un ruolo nel quale il professore di filosofia, più di altri, contribuì a cambiare il volto dell'Ateneo di Bologna tra gli anni Cinquanta e gli anni Sessanta, in un periodo segnato dalla 
contrapposizione politica della guerra fredda. La fermezza, che lo portò a schierarsi su posizioni a volte intransigenti, contraddistinse la sua azione di governo in una difficile e non scontata fase di rilancio dell'Alma Mater bononiensis. Sul piano nazionale la sua opera fu dirimente in alcuni passaggi chiave dello sviluppo del sistema universitario in una fase di necessario cambiamento dell'intero assetto italiano, compreso tra vecchie logiche di apparato e nuove esigenze strutturali.

\section{Tra incarichi locali e nazionali}

Dapprima funzionario amministrativo presso il Ministero della Pubblica istruzione, Battaglia, nato nel 1902 in provincia di Reggio Calabria, inizia la sua carriera universitaria nel 1927 quando ottiene la libera docenza in Filosofia del diritto, materia nella quale diviene nel 1935 professore ordinario a Siena presso la Facoltà di Giurisprudenza. Viene poi chiamato sotto le Due Torri nel 1938 su proposta del preside della Facoltà di Lettere e filosofia Leonardo Bianchi per ricoprire la cattedra di Filosofia morale?

Sulla sua chiamata pesa senza dubbio il ruolo di Giovanni Gentile e la situazione che si viene a creare a Bologna in seguito alla promulgazione delle leggi razziali [Salustri 2009]. La cacciata degli anti-idealisti da parte del fascismo, iniziata dopo i manifesti di Gentile e Croce, viene completata all'interno dell'Università felsinea nel 1938, quando i provvedimenti antisemiti colpiscono nella Facoltà di Lettere Rodolfo Mondolfo, noto storico della filosofia che non aveva risparmiato pubbliche critiche alla riforma gentiliana del 1923 [Pironi (ed.) 2005].

La figura di Battaglia si inserisce in un quadro articolato entro il quale si ridisegnano le discipline filosofiche insegnate a Bologna, un mutamento iniziato con l'arrivo del gentiliano, e già direttore della rivista dell'Università fascista bolognese, Giuseppe Saitta [Salustri 2011], che dopo la chiamata di Battaglia passa alla cattedra di Storia della filosofia [Salustri 2010]. D'altronde quando il futuro rettore arriva all'Alma Mater la sua vicinanza intellettuale a Gentile è fuor di dubbio: il giovane allievo non ha fatto mancare il suo apporto all'Enciclopedia italiana firmando alcune importanti voci politiche di impianto teorico in funzione dell'ideologia del fascismo. Allo studioso, Gentile ha affidato la compilazione di Democrazia, Nazione, Partito e Stato, voci nelle quali il richiamo al Fascismo, redatto dallo stesso Gentile, sono costanti [Turi 2002, 138-44].

ASUB, Professori ordinari, fascicoli individuali (pos. 4/a), Felice Battaglia, Lettera del rettore Alessandro Ghigi, 10 dicembre 1938. 
Eppure a Bologna, Battaglia, che nel 1940 ottiene la direzione del Centro studi di dottrina del fascismo, si fa promotore di un pensiero liberale e libertario che nella pratica lo porta ad essere un maestro di nomi noti dell'antifascismo tra i quali spiccano Vittorio Telmon, Renato Zangheri e Nicola Matteucci; personaggi molto differenti tra loro, a sottolineare il giudizio che Zangheri ci ha offerto del suo maestro, ricordandolo soprattutto per la sua «lezione di tolleranza» [Finzi e Lama 1985, 109-10].

Nei primi giorni successivi alla Liberazione è affidata a Battaglia la guida dell' $\mathrm{Al}$ ma Mater di Bologna e in qualità di commissario egli rimane in carica fino al 5 maggio 1945, quando diviene pro-rettore Edoardo Volterra. La scelta di Battaglia è dovuta alla posizione che egli ha mantenuto durante il periodo dell'occupazione nazista, una «posizione di neutrale isolamento» [Andreoli et al. 1976, 21], ma anche di scontro con l'allora rettore della Repubblica sociale italiana Goffredo Coppola ${ }^{3}$, che permette ai liberatori di affidargli un incarico tanto delicato. L'ex-preside accetta il difficile incarico temporaneo e la fiducia in lui riposta è confermata dal voto unanime della costola felsinea del Comitato di liberazione nazionale intellettuali, del quale Battaglia fa parte sin dal periodo cospirativo in rappresentanza del Partito d'Azione. Lo stesso Comitato si fa promotore di un voto a favore dell'epurazione da condurre «con rigido spirito di legalità»", e questa modalità è quella seguita dalla Commissione epurativa universitaria bolognese che, dopo una rapida valutazione anche sull'attività di Battaglia, ne stralcia la posizione [Salustri 2003, 125-52]. Come detto, alla fine del breve commissariamento, stabiliti i rapporti di forza tra alleati e Comitato di liberazione bolognese, viene nominato pro-rettore Volterra, già indicato per questo compito nel 1944, ancora prima della liberazione della città di Bologna.

I problemi da affrontare sono molteplici. Su scala regionale la prima questione che coinvolge congiuntamente Volterra e Battaglia è relativa ad alcuni corsi ai quali l'Università di Modena non vuole rinunciare, esami e insegnamenti introdotti durante il periodo del governo della Repubblica sociale e giustificati con la situazione degli studenti sfollati, impossibilitati a recarsi nel capoluogo regionale per motivi bellici. I due professori bolognesi accusano l'Ateneo modenese di far sostenere esami in Lettere nella Facoltà di Giurisprudenza e di rilasciare diplomi di laurea in Ingegneria in una Facoltà di Scienze che possiede esclusivamente il biennio matematico. Di conseguenza, chiedono l'intervento del Governo militare

Necrologi, Felice Battaglia, in Annuario dell'Università di Bologna (d'ora in poi AU) 1976-1978, p. 474.

ASUB, Fondo Battaglia, corrispondenza, gennaio-maggio 1945, Relazione del Cln universitario, 22 aprile 1945 allegata a lettera, 6 maggio 1945. 
alleato e del Ministero della Pubblica istruzione. Temono, oltre che la svalutazione dei titoli di studio dell'università in generale, le ripercussioni che la concorrenza da parte di Modena potrebbe avere su Bologna in un periodo critico. L'apertura di corsi, per così dire non ufficiali, in questi anni non affligge certo solo la città delle Due Torri, ma il peso su scala locale di un dualismo "sleale" preoccupa non poco i vertici dell'Ateneo che, seppur distanti da un punto di vista politico, hanno ben chiare le finalità che li uniscono .

Il dopo Liberazione è una proiezione verso il rilancio dell'Ateneo attraverso in primis la ricostruzione, un impegno pressante che porta ad accantonare le differenze. Felice Battaglia, non immune dall'atteggiamento che induce i docenti ad agire nel nome della continuità delle università tra fascismo e dopoguerra ${ }^{6}$, diviene ben presto uno dei punti di riferimento di una ripresa che deve necessariamente muovere da un rinnovato patto con la società civile e con la città di Bologna. Solo così il lavoro intrapreso da Volterra sin dall'aprile 1945, e proseguito ad opera di Guido Guerrini dal 1947 al 1950, può essere portato a compimento, permettendo all'Ateneo di riprendere una crescita interrotta a causa delle enormi distruzioni e perdite subite da istituti, attrezzature e materiale scientifico durante il conflitto [Negrini e Salustri 2010, 105-22].

Nel 1948 Battaglia, mentre si trova alla guida della Facoltà di lettere e filosofia, è chiamato dal ministro Guido Gonella a far parte della sottocommissione dell'istruzione superiore della Commissione nazionale d'inchiesta per la riforma della scuola che nei primi anni di costruzione democratica del paese deve verificare le condizioni dell'istruzione pubblica e privata e proporre una riforma in grado di superare il modello gentiliano e la politica dei ritocchi di Bottai [Gonella 1981; Santamaita 1999]. Non è questa la sede per soffermarci sui lavori della Commissione e sul ruolo di Battaglia, che, chiamato in quanto redattore del periodico "La riforma della scuola", almeno dalla documentazione reperita non sembra essere stato significativo rispetto a quello di altri membri'. In questa sede sembra però utile sottolineare come sin dall'immediato dopoguerra il professore bolognese abbia sempre ricoperto ruoli di carattere nazionale, capace di tessere rapporti in tutti i contesti dell'alta cultura. Sono gli stessi anni in cui entra a far parte del

ASUB, Fondo Battaglia, corrispondenza, giugno-luglio 1945, Lettera di Volterra a Battaglia, 9 giugno 1945; Lettera di Volterra a W.E. Pratt, 7 giugno 1945 e Lettera di Volterra al ministro della Pubblica istruzione, 9 giugno 1945.

Non è un caso se Ugo Spirito scrive a Battaglia per trovare una sponda nel Consiglio superiore della Pubblica istruzione ed impedire l'epurazione di Camillo Pellizzi [Breschi e Longo 2003, 216].

ASUB, Consiglio superiore della Pubblica istruzione (d'ora in poi CSPI), b. 4 (1947-1966), f. 1, Lettera del ministro Gonella a Battaglia, 16 gennaio 1948. 
Consiglio superiore della Pubblica istruzione ${ }^{8}$, mentre nel 1950 è scelto sempre da Gonella per partecipare ai lavori della Consulta didattica nazionale, un altro organismo creato dal ministro per definire il riordino dei programmi scolastici.

Due incarichi nazionali che contribuiscono ad aprire per Battaglia la strada del rettorato dell' Alma Mater bononiensis. A 48 anni, il nuovo rettore viene scelto il 22 ottobre 1950 dal corpo accademico felsineo con 55 voti su 90 votanti, i restanti 34 vanno al rettore uscente Guerrini e un solo voto viene espresso a favore di Gino Bottiglioni'. Con una maggioranza molto simile a quella ottenuta da Guerrini nei confronti del suo predecessore Volterra ${ }^{10}$, Battaglia raccoglie lo scettro in un momento chiave per l'Università di Bologna e per l'intero paese.

\section{I primi anni Cinquanta e la nuova convenzione edilizia}

Nella prima prolusione da rettore pronunciata nel gennaio 1951 dal significativo titolo I doveri dell'Università nell'ora presente, Battaglia si rivolge ai colleghi, agli studenti e alle autorità non per un vero e proprio discorso inaugurale, ma per «un chiarimento interno di reciproci compiti». Il tema è il ruolo del docente, sia esso scienziato o filosofo, chiamato ad una responsabilità senza precedenti nel mondo emerso dalla Seconda guerra mondiale, dove la scienza sembra drammaticamente piegata alla pura tecnica. Il discorso di Battaglia, dal quale emergono la formazione spiritualistica e la valenza cristiana della sua formazione liberale, tocca i nodi chiave della modernità post-bellica e si sposta da un piano teorico ad uno strettamente pratico, affrontando le carenze con le quali l'Università bolognese deve fare i conti per poter rispondere alle nuove questioni che si manifestano al mondo intellettuale"1.

Rispetto al predecessore, che si è rivolto ripetutamente al Ministero della Pubblica istruzione e a quello dei Lavori pubblici, Battaglia indirizza subito la sua azione verso i poteri locali che elegge a interlocutori principali dell'Ateneo, forte di

8 ASUB, Professori ordinari, fascicoli individuali (pos. 4/a), Felice Battaglia, Lettera del rettore Guerrini a Battaglia, 12 febbraio 1948.

ASUB, Rettore (pos. 1), b. 5 (1940-1965), f. Nomina del rettore triennio 1950-1953, Verbale della seduta del corpo accademico, 22 ottobre 1950.

Nella seduta del 1947 Guerrini aveva ottenuto 52 voti contro i 34 di Volterra: ivi, f. Elezione del rettore per il triennio 1947-50, Verbale della seduta del corpo accademico, 29 ottobre 1947.

Inaugurazione dell'anno accademico, 20 gennaio 1952. I doveri dell'Università nell'ora presente. Discorso del Magnifico rettore prof. Felice Battaglia. Relazione per l'anno 19491950, in AU 1950-51/1951-52, pp. 118-126. 
una rinata leadership, anche politica, della figura rettorale. Per rilanciare l'Alma Mater occorre costruire una comunità di intenti con tutta la città così come era stato fino a quel momento, sin dalla prima convenzione firmata alla fine dell'Ottocento, e i tempi sembrano maturi quando la Giunta comunale social-comunista capitanata dal sindaco Giuseppe Dozza si fa carico della «questione universitaria» maturata all'interno del gruppo dirigente comunista. L'attenzione del Partito comunista locale per l'Università trae la sua forza dalla presenza nel Comitato federale dei docenti Paolo Fortunati - rappresentante comunale nel Consiglio di amministrazione dell'Università tra gli iniziatori della prima cellula comunista all'interno dell'Ateneo -, Protogene Veronesi, Luciano Bergonzini e Renato Zangheri, che comprendono l'importanza di individuare la strada per aprire al partito un mondo ancora diffidente verso i comunisti. La questione culturale e i problemi dell'Ateneo vengono posti al centro di un Comitato federale del Pci che si svolge nei primi giorni del giugno 1952 nel quale si auspica una ripresa dei rapporti tra poteri locali e Università e «si stigmatizza[...] il disinteresse della classe politica nazionale nei confronti delle istituzioni culturali» [Lama 2007, 252]. Come è stato giustamente sottolineato, il dibattito risulta carente rispetto ad una visione sull'intero problema universitario che guardi ai mali dell'Università e al suo immobilismo come concause della sua arretratezza [Finzi e Lama 1995, 113]. D'altronde, la posizione locale del Pci nei primi anni Cinquanta rispecchia quanto accade a livello nazionale più o meno per tutti i partiti dell'arco costituente: non si intende considerare la politica universitaria come una priorità del sistema italiano e si lasciano i processi decisionali agli interessi di settore e al ceto accademico, salvo poi intervenire per sanare le situazioni di emergenza o per collocare sterili bandierine ideologiche su singoli temi [Bonini 2007].

La seduta del Comitato federale del Pci bolognese è propedeutica ad un'importante seduta del Consiglio comunale di Bologna che, grazie anche alla spinta propulsiva di Battaglia, imprime un'accelerazione al percorso di rilancio della locale Università. Per riportare l'Ateneo agli antichi splendori o, per dirla meglio con le parole del rettore, per far sì che l'Alma Mater felsinea possa competere sul piano nazionale e internazionale con i più prestigiosi centri di ricerca, occorre puntare sul tessuto territoriale e riannodare i legami con il Comune, interlocutore principe dell'Università ancor prima dello Stato.

Battaglia, che ha vissuto nell'Ateneo gli ultimi anni del rettorato di Ghigi e conosce l'importanza dei soggetti che hanno contribuito alle diverse convenzioni edilizie, è anche consapevole che la situazione che si prospetta all'indomani del dopoguerra è nettamente mutata e si deve far leva sulle nuove istanze politiche. Per questo motivo illustra al Consiglio comunale di Bologna quelle che sono le 
sue preoccupazioni e le possibili soluzioni.

Ad introdurre la relazione di Battaglia è il sindaco Giuseppe Dozza che nella seduta del 30 giugno 1952 si rivolge al rettore e ai consiglieri ricordando l'importanza dell'Università nella storia cittadina e la necessità da parte del Comune, nell'interesse della cittadinanza, di collaborare alla soluzione dei problemi esposti dalla massima autorità accademica. Da parte del sindaco non c'è subalternità; al contrario Dozza apre al discorso del rettore invitandolo a parlare nella sede in cui il Consiglio si raduna, e questo invito è una chiara propensione alla risoluzione dei problemi, che per la Giunta deve avvenire con la più ampia convergenza di tutte le forze politiche al fine di prendere decisioni condivise che peseranno in maniera significativa sul bilancio. Al contempo, riafferma implicitamente l'autonomia rispetto ad un Ateneo il cui il corpo docente guarda con diffidenza alla maggioranza che guida la città.

Il lungo intervento di Battaglia, finalizzato a riallacciare i fili di un dialogo diretto con il Comune, non si rivolge al passato, bensì punta al futuro dell'Ateneo e si mantiene su linee ferme di assoluta autonomia decisionale degli organi universitari. Il rettore non fa sconti e non nasconde la situazione drammatica in cui versa la sua Università. Se i contributi annui dello Stato e degli enti locali sono appena sufficienti per la gestione ordinaria, i problemi che espone Battaglia sono di più lungo periodo e possono essere risolti a partire dagli stessi presupposti che hanno portato alla firma delle convenzioni passate. Una progettualità a dir poco ambiziosa che guarda a tutto tondo al rilancio dell'Ateneo a partire dalla sistemazione degli indirizzi tradizionali, attraverso nuove sedi e ampliamenti funzionali di sedi preesistenti, per giungere a rendere Bologna una sede universitaria attrattiva per gli universitari stranieri, segno distintivo della secolare storia dell'A teneo felsineo. Il discorso si chiude con un riferimento al diritto allo studio e alla giustizia sociale. Battaglia tocca un tema caro alla maggioranza social-comunista e lo fa sgomberando ogni dubbio circa la sua posizione anche rispetto agli scioperi studenteschi ricorrenti sin dal 1950 e finalizzati a contrastare i temuti aumenti delle tasse. Le categorie individuate nel discorso del rettore sono quelle degli abbienti che devono pagare le tasse e dei non abbienti meritevoli che devono ricevere l'assistenza necessaria per poter completare al pari degli altri i propri studi ${ }^{2}$.

Una volta conclusi gli interventi dei consiglieri viene letto l'ordine del giorno, con il quale il Consiglio comunale si impegna a prendere in esame tutte le necessità dell'Ateneo per poter giungere ad una soluzione adeguata nel rispetto dell'autono- 
mia dell'istituzione universitaria. Il sindaco Dozza, riprendendo la parola, afferma come le proposte fatte dal rettore abbiano già avuto «una prima elaborazione da parte della Giunta municipale» e siano già state discusse con Battaglia «fra quattro pareti», sia in merito alle aree sia in merito ai contributi; Dozza dichiara inoltre che, viste le esigenze finanziarie, il Comune deve farsi capofila di un appello da rivolgere agli altri comuni, alle provincie e a tutti gli enti interessati all'Università' ${ }^{3}$. Quando il rettore informa il Senato accademico dei progressi conseguiti in rapporto all'impegno del Comune, i membri dell'organo decidono di votare un ordine del giorno che venga reso pubblico per frenare l'ottimismo, diffusosi anche a causa dell'approvazione della legge che prevede l'aumento dei contributi statali a favore dell'Ateneo ${ }^{14}$. La preoccupazione espressa pubblicamente dal Senato, e condivisa dai direttori di molti Istituti universitari, è provocata dall'aggravarsi della situazione dell'Alma Mater per l'aumento dei costi di gestione ordinaria e straordinaria ed è l'elemento che spinge definitivamente il rettore Battaglia all'azione: la ripresa dell'Università non può prescindere dal territorio e dagli enti che lo governano, siano essi pubblici o privati. Anche considerando i nuovi stanziamenti statali è infatti impossibile provvedere al fabbisogno dell'istituzione. Il successo di Battaglia è completo su ogni fronte e spiana la strada alla sottoscrizione di una nuova convenzione con gli enti locali e con vari enti economici cittadini, oltre che ad una sua 'naturale', o per meglio dire scontata, riconferma alla guida dell'Ateneo per un ulteriore triennio. Nell'ottobre 1953, 84 docenti su 89 votano la rielezione di Battaglia: si tratta di un plebiscito che dimostra la condivisione da parte dei docenti delle scelte politiche intraprese dal rettore, scelte capaci di cancellare le opposizioni che avevano accompagnato la prima elezione ${ }^{15}$. Anche lo scoglio della seduta comunale del gennaio 1953, nella quale il consigliere social-democratico Pietro Crocioni mette in luce una delle criticità maggiori di questo nuovo accordo, vale a dire la "sudditanza" del Comune alle scelte decisionali dell'Università e al suo piano di interventi, viene rapidamente superato in nome di un'autonomia della cultura e dell'insegnamento che trova tutti concordi ${ }^{16}$. La nuova convenzione è siglata formalmente il 25 febbraio 1954. Dopo una parziale modifica viene approvata con decreto del presidente della Repubblica nel novembre 1956; la prima importante realizzazione è la creazione a Bologna della

Ivi, pp. 642-644, le citazioni sono a p. 642.

14 ASUB, Verbali Senato accademico (d'ora in poi VSA), 25 luglio 1952, pp. 108-109.

ASUB, Rettore (pos. 1), b. 5 (1940-1965), f. Elezione del rettore triennio 1953-1956, Verbale della seduta del corpo accademico, 26 ottobre 1953.

ACB 1953, Bologna, tip. L. Parma, 1953, seduta 7 gennaio 1953, p. 9. 
Facoltà di Magistero, sulla base della norma contenuta nel Testo Unico del 1933 che permette la creazione di nuove cattedre in convenzione. Magistero, che non rientrava tra gli indirizzi di studio presenti nell'Università felsinea per motivi politico-istituzionali che ne hanno bloccato la nascita sin dal 1923, viene creata per rispondere alle esigenze di un bacino d'utenza di licenziati magistrali costretti a rivolgersi principalmente all'Ateneo di Firenze per continuare gli studi, un problema ribadito dall'assessore Renato Cenerini di fronte al Consiglio comunale per favorire l'approvazione della convenzione ${ }^{17}$.

Tale esigenza si sposa con la volontà dei docenti di Lettere e filosofia di potenziare gli indirizzi di studio universitari nei quali inserire nuove cattedre e insegnamenti, la cui qualità è garantita dalla stessa Facoltà di origine. Battaglia, prima come preside della Facoltà e poi come rettore, fa sue queste istanze e, in qualità di presidente del Consorzio interprovinciale universitario - che ritorna alla sua funzione originale dopo essere stato impegnato interamente nella ricostruzione -, dà il via libera al nuovo indirizzo di studio che deve la propria origine all'impegno del Consorzio stesso. Il reale riconoscimento come Facoltà arriva solo nel novembre 1956 quando, grazie alla tenacia di Battaglia, di alcuni docenti bolognesi e degli enti coinvolti nel Consorzio, nel frattempo rifinanziato dal Comune di Bologna, si ottiene il decreto presidenziale di istituzione [Preti 2006, 21-35]. Nello scontro tra poteri locali e docenti che spingono per il nuovo indirizzo, appoggiati da esponenti politici bolognesi - tra i quali spicca il consigliere comunale, sottosegretario alla Pubblica istruzione e allora direttore de "il Resto del Carlino" Raimondo Manzini -, e chi si oppone all'istituzione di una nuova Facoltà affiancando a motivazioni di carattere pedagogico logiche di potere e di ripartizione di cattedre su base nazionale, Battaglia gioca ancora un ruolo di primo piano. È il rettore che durante l'inaugurazione dell'anno accademico 1955-56 dà pubblicamente il solenne annuncio della nascita del nuovo indirizzo portando dalla sua parte l'intera opinione pubblica, potendo così premere maggiormente per ottenere il consenso del Consiglio superiore della pubblica istruzione. Ed è lo stesso Battaglia, grazie ad una fitta corrispondenza, a guadagnarsi il voto favorevole del Consiglio anche spingendo il Comune di Bologna ad incrementare il 
proprio intervento nel Consorzio ${ }^{18}$.

Nel frattempo, la nuova sede della Facoltà di Economia e commercio, che ospita la Scuola di Statistica diretta dal consigliere comunale e senatore Paolo Fortunati, è inaugurata il 15 ottobre 1955 alla presenza di Giovanni Gronchi, presidente della Repubblica. Le risorse locali hanno permesso l'affidamento dell'incarico di progettazione all'architetto Luigi Vignali e la nuova sede, costruita attorno ad un portico situato di fronte all'ingresso del rettorato di via Zamboni, diviene presto il simbolo della ripresa edilizia universitaria [Vignali 1952, 7-8]. Il segnale che l'Università vuole riaffermare un suo ruolo anche a livello internazionale arriva invece dall'inaugurazione in via Belmeloro della filiale europea della Johns Hopkins University. Un esperimento di alta formazione che prende vita grazie anche all'intervento diretto di Battaglia e al suo confronto con il professor Groves Haines, propugnatore di questo modello educativo [Evans 1995, 24-6], che saranno motivo di non pochi problemi per il rettore ${ }^{19}$.

\section{La frattura con i comunisti}

Quando nel 1956 la Convenzione viene approvata, anche la proposta elaborata due anni prima da un gruppo di parlamentari locali per la Sistemazione edilizia dell'Università di Bologna diviene legge, stabilendo uno stanziamento di 1.860 milioni di lire - metà di provenienza statale e metà suddivisi tra Comune, Università, Provincia, Camera di Commercio e banche - a favore dell'edilizia universitaria [Finzi e Lama 1995, 114]. I rapporti tra Università e Comune sono oramai consolidati, la forza dei due soggetti non è in discussione, ma gli equilibri sono spezzati dall'azione della Giunta, ancora capitanata da Dozza al secondo mandato, che ribalta il suo atteggiamento rispetto a quello tenuto in occasione della convenzione. Il Comune decide di finanziare l'Istituto universitario di Fisica Righi con un contributo diretto di 500 milioni di lire al fine di condurre ricerche sull'energia nucleare e la sua applicazione pacifica.

Ad aprire i lavori del Consiglio comunale nel febbraio 1956, è infatti il professor Giampietro Puppi, fisico delle particelle elementari e direttore dell'Istituto di Fisica, non più il rettore, che non è presente alla seduta. L'assenza di Battaglia non è certo casuale e si lega con molta probabilità alla fine del suo secondo mandato.

Inaugurazione dell'anno accademico 1955-56, 9 gennaio 1956, Relazione del magnifico rettore prof. Felice Battaglia per l'anno 1954-55, in AU 1954-55/1955-56, pp. 273-274.

19 ASUB, VSA, 30 settembre 1954, p. 233. 
La Giunta decide di forzare i rigidi schemi entro i quali erano stati gestiti i rapporti con l'Università sin dagli inizi del Novecento, puntando su uno sviluppo scientifico che esca dai confini locali per attingere ad una prospettiva europea. $\mathrm{Si}$ tratta di un impegno di spesa che vede la componente comunista alla guida della città farsi promotrice di investimenti che guardano al progresso tecnologico dei futuri dieci anni, capaci di rendere l'Istituto bolognese - come lo stesso Puppi afferma nel suo intervento - indipendente dalla politica statale nei riguardi della ricerca. Il programma presentato dal direttore, elaborato alla luce dei gravi ritardi del paese nel settore scientifico, non è a breve scadenza e prevede importanti investimenti (50 milioni l'anno) [Lama 1987, 249] finalizzati non solo a comprare macchinari, ma a «fare una scuola, per produrre nuove leve, per soddisfare alle richieste sempre crescenti del Paese $»^{20}$.

Il rettore sembra lontano da questa nuova campagna, nella quale dovrebbe essere impegnato in prima linea; sono infatti passati i giorni di piena corrispondenza con la classe politica bolognese social-comunista. Sul finire del 1954 il magnifico ha aperto una frattura difficile da risanare opponendosi alla chiamata da parte dell'Ateneo di Ferruccio Parri in qualità di oratore ufficiale alla commemorazione della battaglia dell'Università. Il 20 ottobre, come ogni anno dal 1944, l'Alma Mater felsinea commemora gli antifascisti uccisi all'interno delle proprie mura da reparti delle SS e dalle Brigate nere e lo fa con una cerimonia ufficiale [Brizzi (ed.) 2005, 15-52]. Nel decennale si forma un comitato di cui fanno parte expartigiani, docenti universitari, un assessore e le consorti di due resistenti uccisi ${ }^{21}$. Nella seduta del Senato accademico del 30 settembre Walter Bigiavi propone di invitare come oratore ufficiale Ferruccio Parri in qualità di «capo della Resistenza»; dopo una breve discussione il Senato decide di chiamare alla commemorazione i professori che hanno preso parte alla lotta di Liberazione e di conferire in quell'occasione le lauree ad honorem agli studenti caduti. Battaglia, che accetta di buon grado il mandato per organizzare l'evento, propone una terna diversa di oratori $^{2}$. La discussione riprende nella seduta successiva, il 7 ottobre, quando il rettore informa i colleghi di aver ricevuto il comitato per le commemorazioni che ha riproposto Ferruccio Parri in sintonia con la richiesta di Bigiavi. È quest'ultimo in Senato a farsi nuovamente carico della scelta di Parri come oratore ufficiale della cerimonia, indicato non solo perché a capo della Resistenza, ma in quanto

20 ACB 1956, vol. I, Minerbio, Soc. Bevilacqua-Lombardini, 1956, seduta 28 febbraio 1956, p. 245.

21 ASUB, VSA, 7 ottobre 1954, pp. 237-238.

22 Ivi, 30 settembre 1954, pp. 232-233. 
presidente del Consiglio dopo la Liberazione. Purtroppo i brevi resoconti delle sedute del Senato accademico non riportano in dettaglio la discussione, quello che traspare da questa fonte è solo il risultato finale. Dai verbali emerge che dopo l'intervento di tutti i presidi, sei voti favorevoli, un'astensione e il voto contrario di Bigiavi, si stabilisce che le modalità di svolgimento della commemorazione debbano essere decise dall'Università e che sarà il rettore a scegliere l'oratore ufficiale tra i docenti dell'Ateneo o di altra sede ${ }^{23}$; a nulla valgono le successive proteste del comitato.

Il muro alzato dal Senato e da Battaglia porta all'immediato scontro con il Partito comunista. Dalle pagine di "Emilia", mensile del partito, parte un duro attacco all'Ateneo. Nel numero di novembre 1954 l'editoriale è dedicato all'Università di Bologna e riporta nel dettaglio quanto sembra essere accaduto in Senato accademico: «c'è stato chi non ha avuto vergogna di proporre, come condizione e corrispettivo alla celebrazione degli eroici Caduti partigiani, quella dei loro aguzzini fascisti, che "sono caduti anche loro"». L'articolo prosegue criticando l'operato del Senato, ritenuto «meschino e indegno [per] rappresentanti elevati della cultura italiana» che hanno anche rifiutato Calamandrei. L'umiliazione sarebbe ancora maggiore - chiosa l'articolo - se, insistendo sul nome di Parri, il capo della Resistenza «si presentasse, chiamato dalla città, a celebrare in altra sede, quegli episodi. Se l'Università di Bologna insistesse nel respingerlo, essa creerebbe un solco grave fra sé e l'opinione pubblica cittadina, fra sé e le sue stesse tradizioni migliori $»^{24}$.

La risposta del rettore è altrettanto forte ed è diretta in prima persona verso quei docenti che hanno preso parte alla denuncia. Tra le carte di Veronesi conservate presso la Fondazione Gramsci Emilia-Romagna abbiamo potuto rintracciare copia della lettera inviata a Protogene Veronesi, redattore di "Emilia", firmata da Battaglia che si va ad affiancare a quelle recapitate a Luciano Bergonzini, allora condirettore della rivista e membro del comitato, e a Carlo Volpe, che sarà spinto a distaccarsi da "Emilia" [Finzi e Lama 1995, 117]. Assistenti universitari, questi, che ricevono una dura ammonizione da parte del rettore per aver osato sfidare le gerarchie accademiche. Il linguaggio usato da Battaglia è molto chiaro ed evidenzia i tratti di un richiamo fondato su due elementi che riassumono al contempo la situazione di scontro politico presente anche all'interno dell'Ateneo bolognese e la riaffermazione da parte del più alto rappresentante dell'Università di una concezione del contesto accademico come di un mondo all'interno del quale vigono

Ivi, 7 ottobre 1954, pp. 237-238.

Aula Magna, in "Emilia", 33, novembre 1954, 337-338. 
regole ben definite che devono essere rispettate da chi entra a farne parte. Il rettore accusa i redattori della rivista di essersi «lasciati andare a raccogliere indiscrezioni sulla discussione» svolta in seno al Senato accademico sulle celebrazioni e «ad esprimere nei confronti del nostro più alto organo accademico parole che io non posso che ritenere inopportune, se non addirittura ingiuriose». Nel rivolgersi direttamente a Veronesi, Battaglia gli ricorda il suo ruolo di assistente universitario che dovrebbe avere a cuore «l'indipendenza dell'Università» come tutti coloro i quali vivono al suo interno. Il comportamento deprecabile per il rettore non è dunque il dissenso politico, definito «sempre lecito», ma «un biasimo che vorrebbe toccare la stessa moralità dei docenti illustri che hanno l'onore di sedere nel Senato accademico». Battaglia punta quindi sull'indipendenza dell'Ateneo e dei suoi organi e si dice impossibilitato a nascondere «la gravità» di quanto accaduto, soprattutto perché l'attacco proviene dall'interno dell'Università?'.

La polemica non si stempera con il passare dei mesi e arriva alle pagine nazionali de "l'Unità", dopo che, il 17 aprile 1955, Parri chiude con un suo discorso le celebrazioni nazionali per il decennale della Resistenza tenutesi a Torino ${ }^{26}$. Il giorno successivo, in un articolo dal titolo Frati volanti e polizia contro la cultura a Bologna che disegna i tratti di una regione nella quale lo scontro ideologico sembra coinvolgere tutte le espressioni della cultura, Battaglia viene equiparato al rettore di Parma che vuole concedere la laurea alla memoria ad un milite di Salò. Le due guide degli Atenei regionali sono accusate di far parte «di un piano concertato allo scopo di rivalutare il fascismo», un piano che passa attraverso la creazione della Johns Hopkins University, un centro che esiste «soltanto nei paesi coloniali», il divieto di portare in scena le commedie di Tennessee Williams, il controllo e le denunce dei frati volanti, dove non arriva la celere con i suoi divieti, e il «minacciare di provvedimenti disciplinari (con garbo s'intende) gli stessi professori dell'Ateneo $»^{27}$.

Al di là del tema specifico, lo scontro che si consuma racchiude in sé tutti gli elementi di tensione e di distensione che contraddistinguono la politica nazionale - segnata dall'accordo raggiunto anche con i democristiani per l'elezione di Giovanni Gronchi alla presidenza della Repubblica - e internazionale con l'apice dei fatti di Ungheria, ma anche l'inizio di una diversa fase di rapporti tra Ateneo e città. Lo scontro Dozza-Dossetti alle amministrative e la terza vittoria del sin-

Fondazione Gramsci Emilia-Romagna, Archivio di Protogene Veronesi 1943-1990, Documenti personali e biografici 1943-1959, Carteggio 1943-1958, riservata-personale, 16 marzo 1955.

26 II convegno del decennale della Liberazione, in "I'Unità", 17 aprile 1955, p. 1.

27 Frati volanti e polizia contro la cultura a Bologna, ivi, 18 aprile 1955, p. 7. 
daco comunista aprono la strada a quel piano di "buona amministrazione" che da manifesto elettorale diviene pilastro della politica cittadina, segnata da una forte presenza di professori universitari nelle liste elettorali e poi nella Giunta [Fanti e Ferri 2001, 35-6; Lama 2007, 278-80] $]^{28}$; contemporaneamente, anche la lista di Dossetti guarda con attenzione al mondo universitario, e non solo a quello felsineo [Tesini 1986, 151]. Di fronte al sempre più forte intreccio politico tra città e Ateneo, e alle scelte di due figure predominanti come Dozza e Dossetti che, seppur da prospettive diametralmente opposte, guardano al futuro cittadino e a quello universitario come a due elementi inscindibili, la gestione dell'Università da parte di Felice Battaglia in questa fase sembra quasi anacronistica. L'interesse della politica cittadina verso l'Ateneo sarà in costante aumento almeno fino alla metà degli anni Sessanta, come bene dimostrano i bilanci comunali e gli atti del Senato, dove molte sono le tracce del finanziamento diretto da parte del governo locale di cattedre e di nuovi istituti universitari [Lama 1987, 189-94]. L'amministrazione diviene così la prima propositrice di investimenti e non più semplice destinataria delle richieste provenienti dall'Università, ma sarà un altro rettore a farsi carico delle nuove convenzioni.

Alle elezioni per il rettorato - triennio 1956-59 - Battaglia riceve un solo voto contro i 74 ottenuti da Giuseppe Gherardo Forni, preside della Facoltà di Medicina e chirurgia e ordinario di Clinica Chirurgica. Una votazione che non stupisce se teniamo conto della lettera messa agli atti e inviata da Battaglia agli aventi diritto al voto pochi giorni prima della seduta del corpo accademico. Nella missiva Battaglia chiede ai colleghi di non essere ricandidato perché interessato «a ritornare più intensamente ai miei studi $\mathrm{e}$ [per] la necessità di un certo riposo dopo lunghe fatiche $)^{29}$. Poche righe accompagnate da un ringraziamento che rappresentano un ritiro temporaneo dal ruolo di guida dell'Ateneo, ma che non si traducono in un impegno ridotto sul piano nazionale dove Battaglia è pienamente coinvolto nel dibattito sul riordino dell'assetto universitario italiano.

\section{Al Consiglio superiore della Pubblica istruzione}

Nelle votazioni per il Consiglio superiore della Pubblica istruzione per il qua-

Nella lista del sindaco uscente, che guadagna il 5\% in più dei consensi rispetto al 1951, vi sono ben 7 docenti; la stessa lista trova l'appoggio di altri nomi importanti della cultura nazionale.

29 ASUB, Rettore (pos. 1), b. 6 (1956-1962), f. Elezione del rettore triennio accademico 1956-1959, Verbale della seduta del corpo accademico, 25 ottobre 1956 e Lettera a tutti i membri del Corpo Accademico dell'Università degli studi di Bologna, 22 ottobre 1956. 
driennio 1958-62 Battaglia è eletto nuovamente come membro della prima sezione con altri tre docenti rispettivamente di Roma, Napoli e Torino in rappresentanza delle Facoltà di Lettere e filosofia, Magistero, Lingue e letterature straniere, dell'Istituto superiore di Economia e commercio di Venezia e dell'Istituto orientale di Napoli; entra poi a far parte della Corte di disciplina del Consiglio ${ }^{30}$.

L'attività, come testimonia la sua corrispondenza, è costante e lo vede coinvolto in un momento cruciale per lo sviluppo delle università italiane. Ricordiamo che il Consiglio, la cui articolazione è normata nel $1947 \mathrm{e}$ in parte modificata dopo dieci anni, si configura come organo consultivo del Ministero della Pubblica istruzione ${ }^{31}$. I pareri del Consiglio non hanno mai valore vincolante per il ministro, salvo alcune eccezioni previste dalla legge, a volte sono ridotti alla sola legittimità di atti o procedimenti, in alcuni casi vengono estesi al merito amministrativo. Ciò non toglie che negli anni Cinquanta e Sessanta il Consiglio si trovi ad affrontare da attore principale i nodi chiave legati allo sviluppo della società italiana, investita da una crescita tale da far pensare ad un imminente deficit numerico nella formazione di quadri con la conseguente necessità di riformare il sistema universitario italiano, anche in funzione di un aumento massiccio della scolarizzazione secondaria. Simili temi sono discussi a Bologna nell'aprile 1960 in un convegno nazionale interamente dedicato alla questione universitaria, che Battaglia presiede nella seduta inaugurale. Il suo discorso, successivo a quello del presidente della Repubblica, pone al centro la crisi che l'università sta affrontando, schiacciata tra «l'accesso delle classi subalterne alla cultura e alle professioni [e] la ricerca nella connessione con la tecnica» e la necessità di trovare soluzioni che non abbassino il livello dell'alta istruzione «distrugge[ndo] quelle selezioni che hanno costituito nei secoli l'efficacia dell'insegnamento universitario; come, d'altra parte, provvedere alla selezione in senso esclusivistico può costituire un odioso atto di ingiustizia sociale di chi fiducioso nel suo ingegno si vede immotivatamente respinto». L'equilibrio tra le due opzioni è la strada indicata da Battaglia [Comitato di studio dei problemi dell'università italiana 1962,

\footnotetext{
30 Archivo centrale dello Stato, Ministero della Pubblica istruzione, Consiglio superiore della Pubblica istruzione (d'ora in poi ACS, MPI, CSPI), Atti, III serie, b. 155, f. 368, Decreto del ministro segretario di Stato per la Pubblica istruzione, 26 novembre 1958 e Estratto del processo verbale dell'adunata, 11 dicembre 1958.

31 I 67 membri, poi divenuti 70 nel 1957, oltre al ministro presidente, sono suddivisi in tre sezioni (la prima per l'istruzione superiore, la seconda per l'istruzione secondaria e la terza per quella elementare): Legge 30 dicembre 1947, $n$. 1477, modificata con legge 2 agosto 1957, n. 699, concernente il riordinamento dei Corpi consultivi del Ministero della P.I., Roma, Istituto Poligrafico dello Stato, 1958. Non esiste ad oggi uno studio che approfondisca le vicende del Consiglio superiore nel periodo del primo dopoguerra paragonabile al lavoro di Ciampi e Santangeli (eds), 1994.
} 
7] che, nelle conclusioni alla tre giorni di studio, sottolinea alcuni punti emersi nel dibattito, quali l'importanza di incentivare un processo di rinnovamento e di democratizzazione di tutta l'istruzione italiana a partire dalla scuola primaria dove va abbandonata la superiorità del latino e del liceo classico per l'accesso all'università, l'esigenza di favorire una maggiore integrazione degli studenti lavoratori e una nuova organizzazione più funzionale fondata sui dipartimenti, punti di collegamento tra atenei e singole cattedre. A questi elementi si aggiunge l'incentivo all'integrazione delle università nel contesto economico locale, nazionale e internazionale, poiché gli atenei devono essere considerati come centri di ricerca non fini a se stessi, ma funzionali allo sviluppo del paese. A tal fine, il professore bolognese sottolinea come nel convegno si sia discusso dell'importanza del ruolo dei Consigli di amministrazione. Infine, nelle conclusioni, Battaglia pone l'accento su altri due temi di fondamentale importanza: gli studenti e i ruoli accademici, a dimostrazione di un dibattito vivo che complessivamente tocca tutti gli aspetti centrali della vita universitaria. Gli studenti, che non vanno considerati come mero oggetto della vita universitaria, ma vanno stimolati nella «responsabilità nella vita universitaria». Infine, a proposito dell'incremento nel numero degli assistenti, dei tecnici e dei professori aggregati ${ }^{32}$, il focus ritorna sulla riorganizzazione interna in dipartimenti e su una maggiore collaborazione tra istituti che superi le logiche ristrette di dominio di singoli docenti e si allarghi fino a costituire una rete di relazioni tra cattedre, dove assuma maggiore valore la selezione a carattere nazionale anche degli assistenti [Comitato di studio dei problemi dell'università italiana 1962, 436].

I nodi chiave della riorganizzazione universitaria sono presentati nel convegno bolognese anche attraverso un documento conclusivo molto articolato che, se non può che essere una sintesi propositiva, elenca comunque in dieci punti quelli che sono gli aspetti sui quali si incentra la discussione sul futuro assetto universitario italiano. Ne emerge sicuramente un tema centrale del quale si occupa nello stesso anno il Consiglio superiore, dietro pressanti sollecitazioni di alcune regioni e località, la cui trattazione vede Battaglia come protagonista. Si tratta del processo decisionale legato al nuovo Piano di distribuzione territoriale delle università elaborato da una commissione specifica la cui relazione finale è presentata proprio 
dal professore felsineo ${ }^{33}$. La posizione di Battaglia emerge sin dalle prime riunioni della Commissione. Come altri commissari egli teme infatti che l'istituzione di nuove sedi universitarie possa incidere sul funzionamento di quelle già esistenti e di fronte alla scelta di creare nuove Facoltà o nuove Scuole, fondare collegi per andare incontro ai fuori sede o una soluzione che tenga presente entrambe le opzioni, ritiene non indispensabile la fondazione di nuove università ${ }^{34}$. La posizione assunta dalla prima Commissione si fonda su una ricca mole di dati statistici che, forniti ai singoli membri dal prof. Tommaso Salvemini, sono poi riassunti negli allegati alla relazione, senza dimenticare il riferimento costante alla fallimentare indagine dell'Associazione per lo sviluppo dell'industria nel Mezzogiorno sulle previsioni relative al mercato del lavoro nel quindicennio 1960-75. I dati elaborati sul sovraffollamento, sull'incremento del numero degli studenti universitari di diversi paesi, e sui laureati in rapporto alla loro residenza sono funzionali a dimostrare che solo per alcune sedi, nello specifico Roma e Napoli, e per alcune Facoltà si possa parlare di sovraffollamento. Pertanto la Commissione sposta la propria attenzione sull'opportunità di intervenire per consolidare le sedi maggiori attraverso «acquisti di locali, di materiale, di personale assistente e docente» ed accrescere la specializzazione delle università minori che non devono continuare a procedere a «scartamento ridotto» come "laureifici". Nella relazione sono comunque presenti aperture verso specifiche aree geografiche dove si devono concentrare i maggiori sforzi di sviluppo. Si tratta del Trentino-Alto Adige che, data la sua complessità e le implicazioni anche internazionali, per Battaglia non può essere affrontato da una semplice consulenza, degli Abruzzi e della regione calabro-lucana. Anche in merito all'istituzione di una nuova sede in Calabria la Commissione preferisce però consigliare al ministro la strada di colleges residenziali ${ }^{35}$.

Cecità della Commissione? Incapacità da parte di Battaglia di comprendere ciò che sta accadendo nel paese e gli indirizzi politici esistenti che mirano ad esempio all'apertura di nuove università, in modo particolare nel Mezzogiorno?

Ovviamente si tratta di domande retoriche. Dalla lettura delle migliaia di carte conservate nell'Archivio storico dell'Università di Bologna relative a Battaglia

Sul dibattito apertosi in merito alle nuove sedi, sul peso del Consiglio superiore e sul ruolo di Battaglia in una realtà specifica si vedano i saggi raccolti in Blanco, Giorgi e Mineo (eds) 2011.

34 ACS, MPI, CSPI, Atti, III serie, b. 190, f. 422, Adunanze, 5 aprile, 12 e 13 maggio 1960.

Ivi, Allegati alla Relazione del consigliere prof. Battaglia sul piano di distribuzione territoriale delle università, le citazioni sono a p. 7. La relazione senza allegati è presente anche in ASUB, CSPI, b. 9 (1956-1961), f. 3 . 
emerge in maniera chiara ed inequivocabile il ruolo avuto dal professore nelle singole vicende universitarie, come dimostra, a ad esempio, un caso specifico come quello della parificazione delle nuove Facoltà dell'Aquila. Nei passaggi chiave di questa evoluzione, che porta nel 1964 alla creazione della libera Università degli Studi dell'Aquila con le Facoltà di Magistero, Medicina e chirurgia, Ingegneria e Scienze matematiche, fisiche e naturali, Battaglia è il referente al quale si rivolge il professore e deputato democristiano Vincenzo Rivera per accelerare le pratiche del riconoscimento ${ }^{36}$. Si potrebbero fare molti altri esempi. Quello che è certo è che Battaglia rimane uomo cardine dei processi che per circa un ventennio segnano l'evoluzione dell'università italiana, come ben evidenzia il ruolo ancora centrale assunto dal professore bolognese, di nuovo rettore, nel lavoro svolto a partire dal 1962 dalle sezioni del Consiglio superiore in merito allo stato e allo sviluppo della Pubblica istruzione italiana ${ }^{37}$.

\section{Un nuovo incarico da rettore}

Battaglia torna alla guida dell'Ateneo proprio nel 1962 quando Forni, ormai fuori ruolo per limiti di età, non può nuovamente essere candidato. La rielezione di Battaglia non è certo cosa facile, come dimostra la prima votazione del corpo accademico che divide equamente i propri voti tra l'ex-rettore e Paolo Dore, 50 contro 50, mentre 21 votanti puntano sulla figura di Tito Carnacini. Battaglia la spunta per 4 consensi nella seconda votazione, mentre molto più semplice è la sua rielezione nel $1965^{38}$.

La nuova stagione rettorale di Battaglia, alla quale vale la pena riservare uno studio specifico che esula dai limiti del presente contributo, si caratterizza in larga parte per la continuità nella crescita dell'Ateneo, perseguita anche da Forni. I tempi sono però nettamente mutati rispetto ai suoi primi mandati: l'università degli anni Sessanta non è più il fulcro della ricerca nazionale e il legame centroperiferia viene ridisegnato in relazione ai nuovi rapporti politici nazionali.

Sono gli anni in cui la mancata volontà politica di giungere ad una riforma organica dell'università finisce per scontrarsi con la contestazione studentesca che

\footnotetext{
36 ASUB, CSPI, b. 8 (1954-1966), f. Carte sciolte, Lettera di Rivera a Battaglia, 17 gennaio 1963.

37 Importanti indicazioni si trovano ivi, f. 7.

38 ASUB, Rettore (pos. 1), b. 7 (1962-1968), f. Elezione rettore triennio 1962-1965, Verbale della seduta del corpo accademico, 20 ottobre 1962 e f. Nomina del rettore per il triennio accademico 1965-66 1967-68, Verbale della seduta del corpo accademico, 19 ottobre 1965.
} 
contribuisce a restringere gli spazi per un vero riformismo dell'intero sistema universitario, complice l'arroccamento del mondo accademico che non vuole e, in parte, non riesce a comprendere la spinta propulsiva della protesta.

Felice Battaglia non è però chiamato ad affrontare il periodo segnato dal 1968: nel marzo decide infatti di dimettersi dalla sua carica per motivi di salute e pochi mesi dopo viene sostituito dal professore più anziano del corpo accademico, Walter Bigiavi, che traghetta per qualche mese l'Ateneo verso le nuove elezioni al rettorato ${ }^{39}$. A Bologna si apre la strada per Carnacini.

\section{Bibliografia}

Andreoli A.M. et al. 1976, Crisi della cultura e dialettica delle idee, IV L'Emilia Romagna nella guerra di liberazione, Bari: De Donato Editore.

Comitato di studio dei problemi dell'università italiana 1962, Studi sull'università italia$n a, \mathrm{~V}$ Una politica per l'università, Bologna: il Mulino.

Blanco L., Giorgi A. e Mineo L. (eds.) 2011, Costruire un'Università. Le fonti documentarie per la storia dell'Università degli studi di Trento (1962-1972), Bologna: il Mulino.

Bonini F. 2007, La politica universitaria nell'Italia Repubblicana, in Brizzi G.P., Del Negro P. e Romano A. (eds.) 2007.

Breschi D. e Longo G. 2003, Camillo Pellizzi: la ricerca delle élites tra politica e sociologia (1896-1979), Soveria Mannelli: Rubbettino editore.

Brizzi G.P. (ed.) 2005, Studenti per la democrazia: la rivolta dei giovani contro il nazifascismo Bologna: Clueb.

Brizzi G.P., Del Negro P. e Romano A. (eds.) 2007, Storia delle Università in Italia, I, Messina: Sicania.

Ciampi G. e Santangeli C. (ed.) 1994, Il Consiglio superiore della pubblica istruzione 1847-1928, Archivio centrale dello Stato, Fonti per la storia della Scuola, II, Roma: Istituto Poligrafico dello Stato.

Ciancabilla L. (ed.) 2010, Bologna in guerra. La città, i monumenti, i rifugi antiaerei, Bologna: Minerva edizioni.

Comitato di studio dei problemi dell'università italiana 1962, Studi sull'università italia$n a$, V Una politica per l'università, Bologna: il Mulino.

Evans R. 1995, Johns Hopkins e Alma Mater, 40 anni di collaborazione, "Saecularia Nona. Università di Bologna, 1088-1988”, 12.

Fanti G. e Ferri G.C. 2001, Cronache dell'Emilia rossa. L'impossibile riformismo del Pci, 
Bologna: Pendragon.

Finzi R. e Lama L. 1995, Comune e Università a Bologna negli anni Cinquanta, in Giuseppe Dozza a dieci anni dalla morte. Dalla lotta antifascista al governo delle sinistre, Bologna Palazzo d'Accursio Sala del Consiglio comunale 15-16 dicembre 1984, Bologna: Graficoop.

Frabboni F. et al. (eds.) 2006, Da Magistero a Scienze della formazione. Cinquant'anni di una Facoltà innovativa dell'Ateneo bolognese, Bologna: Clueb.

Galimi V. e Procacci G. (eds.) 2009, Per la difesa della razza. L’applicazione delle leggi antiebraiche nelle università italiane, Milano: Unicopli.

Gonella G. 1981, Cinque anni al Ministero della Pubblica Istruzione. La rinascita della scuola dopo la seconda guerra mondiale, Milano: Giuffré.

Lama L. 1987, Comune, Provincia, Università. Le convenzioni a Bologna fra Enti Locali e Ateneo (1877-1970), Bologna: Comune di Bologna-Istituto per la storia di Bologna.

Lama L. 2007, Giuseppe Dozza. Storia di un sindaco comunista, Reggio Emilia: Aliberti editore.

Marchello G. (ed) 1953, Felice Battaglia, Torino: Edizioni di Filosofia.

Matteucci N. 1977-78, Felice Battaglia, filosofo della pratica, “Atti della Accademia delle scienze dell'Istituto di Bologna", LXXII, Rendiconti, LXVI.

Negrini D. e Salustri S. 2010, L'Università di Bologna durante la guerra. Distruzione e tutela del patrimonio, in Ciancabilla L. (ed.) 2010.

Novarese D. (ed.) 2011, Accademie e Scuole. Istituzioni, luoghi, personaggi, immagini della cultura e del potere, Milano: Giuffré.

Pironi T. (ed.) 2005, Rodolfo Mondolfo. Educazione e socialismo. Scritti sulla riforma scolastica (dagli inizi del '900 alla Riforma Gentile), Manduria-Bari-Roma: Lacaita.

Polato F. 1988, Battaglia Felice, in Dizionario Biografico degli italiani, 34 .

Polato F. 1989, Bibliografia degli scritti di e su Felice Battaglia, Bologna: Clueb.

Preti A. 2006, Alle origini della Facoltà, in Frabboni F. et al. (eds.) 2006.

Salustri S. 2003, Università e defascistizzazione. Il caso dell'Ateneo di Bologna, "Storia e Problemi Contemporanei”, 32.

Salustri S. 2009, L'Ateneo bolognese e la politica della razza, in Galimi V. e Procacci G. (eds.) 2009.

Salustri S. 2010, Un ateneo in camicia nera. L'Università di Bologna negli anni del fascismo, Roma: Carocci editore.

Salustri S. 2011, L'Università Fascista di Bologna: un modello di accademia per il regi$m e$ ?, in Novarese D. (ed.) 2011.

Santamaita S. 1999, Storia della scuola. Dalla scuola al sistema formativo, Milano: Mondadori.

Scerbo A. 1990, Felice Battaglia: la centralità del valore giuridico, Napoli: Esi. 
Tesini M. 1986, Oltre la città rossa. L'alternativa mancata di Dossetti a Bologna (19561958), Bologna: il Mulino.

Turi G. 2002, Il mecenate, il filosofo e il gesuita. L'Enciclopedia italiana, specchio della nazione, Bologna: il Mulino.

Vignali L. 1952, La nuova sede della facoltà di economia e commercio è il più notevole complesso edilizio realizzato dall'Università degli Studi di Bologna in questo dopoguerra, "Bologna. Rivista del Comune", 8. 